\title{
COMPUTER SIMULATION OF A PLASMA VIBRATOR ANTENNA
}

\author{
Nikolay N. Bogachev ${ }^{a, *}$, Irina L. Bogdankevich ${ }^{b}$, Namik G. Gusein-Zade $^{b}$, \\ VLADIMIR P. TARAKANOV ${ }^{b}$ \\ ${ }^{a}$ Moscow State Technical University of Radio engineering, Electronics and Automation, Moscow, Russia \\ ${ }^{b}$ Prokhorov General Physics Institute RAS, Moscow, Russia \\ * corresponding author: bgniknik@yandex.ru
}

\begin{abstract}
The use of new plasma technologies in antenna technology is widely discussed nowadays. The plasma antenna must receive and transmit signals in the frequency range of a transceiver. Many experiments have been carried out with plasma antennas to transmit and receive signals. Due to lack of experimental data and because experiments are difficult to carry out, there is a need for computer (numerical) modeling to calculate the parameters and characteristics of antennas, and to verify the parameters for future studies. Our study has modeled plasma vibrator (dipole) antennas (PDA) and metal vibrator (dipole) antennas (MDA), and has calculated the characteristics of PDAs and MDAs in the full KARAT electro-code. The correctness of the modeling has been tested by calculating a metal antenna using the MMANA program.
\end{abstract}

KEYWORDS: computer simulation, plasma antenna, KARAT code, MMANA program.

\section{INTRODUCTION}

Many analytical and experimental studies of plasma antennas have been made in recent years [1, 3, 4. These studies have investigated problems of the formation and excitation of plasma by an RF field, transmission and receiving of TEM-waves by plasma antennas, the efficiency of plasma antennas, etc. A group of scientists from the Prokhorov General Physics Institute, Russian Academy of Sciences has made two fairly comprehensive studies [3, 4]. The most promising approach is the use of antennas with discharge plasma - asymmetric dipole length $l=\lambda / 4$ (see Fig. 1).

It is not always possible to investigate plasma antennas with analytical or experimental methods. The best available option is to use computer (numerical) models of plasma antennas. Thin metal vibrators can easily be calculated theoretically in an approximation to an ideal thin vibrator. Plasma dipole antennas with $R \approx 1 \mathrm{~cm}$ cannot be considered when approximating an ideal thin vibrator. For the reasons mentioned above, and due to the features of plasma antennas (gas ionization and excitation of plasma), computer simulation is necessary.

\section{Simulation}

Modern free software for simulating metal antennas does not allow us to establish a plasma environment. In particular, the MMANA software package [2] is convenient for modeling metal antennas of various configurations (stationary mode). Researchers are therefore aiming to design a plasma antenna of finite thickness that transmits a modulated pulse of finite duration.
The KARAT electro-code [5] was chosen as the software for modeling plasma antennas. The KARAT code allows us to simulate a metal antenna and a plasma antenna, and the transmission or receiving of a modulated pulse of finite duration. The KARAT code solves the Maxwell equations using the finitedifference time domain. The material equations of environments may be represented by various models by both phenomenological and PIC (Particle-in-Cell) methods. In our studies, the plasma model is phenomenological.

In the simulations, we modeled the injection of a TEM wave with the frequency through a ring window $(r=1 \mathrm{~cm}, R=2 \mathrm{~cm})$ at the left boundary $z=0$ of the coaxial line. This window is one of the elements of the computation region that is chosen to be the quadrant shown in Fig. 2. The other elements in the computation region are the short coaxial line $5 \mathrm{~cm}$ in length, a radiation output window at the end of the coaxial line, and a finite spatial zone surrounding the plasma column. The outer wall of the coaxial waveguide is adjacent to the transverse conducting screen in the form of a disc.

The correctness of the modeling is confirmed by a comparison ofthe metal antenna results using the KARAT code and using the MMANA program. The calculations of the radiation pattern (RP) for a metal antenna of length $l=17 \mathrm{~cm}$ and a TEM wave with frequency $f=400 \mathrm{MHz}$ show that the two models are quantatively similar (see Fig. 3). 


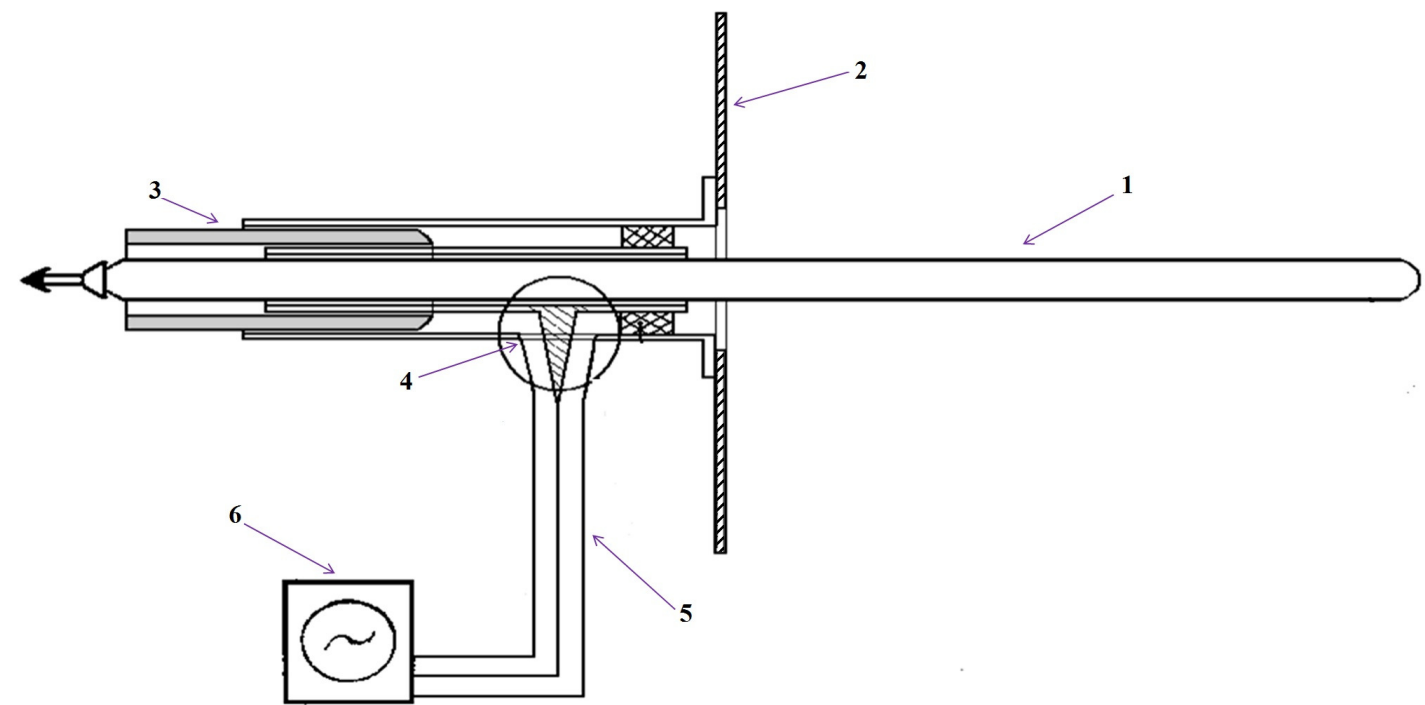

Figure 1. Scheme of the experimental device; 1 - dielectric tube with plasma, 2 - metal screen, 3 - coaxial line, 4 - coaxial tee, 5 - coaxial cable, 6 - transmitter.

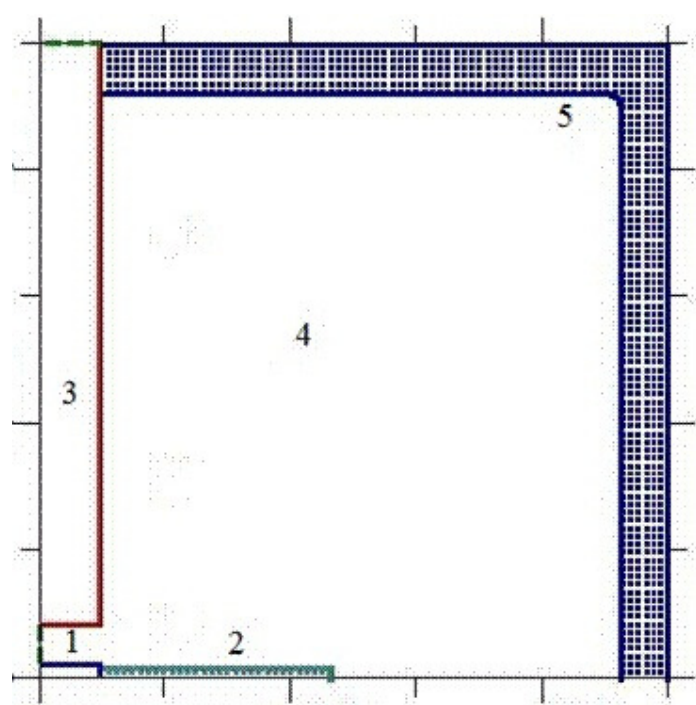

FiguRE 2. Scheme of the computer model in KARAT code: 1 - coaxial cable, 2 - plasma antenna, 3 - metal screen, 5 - absorber.

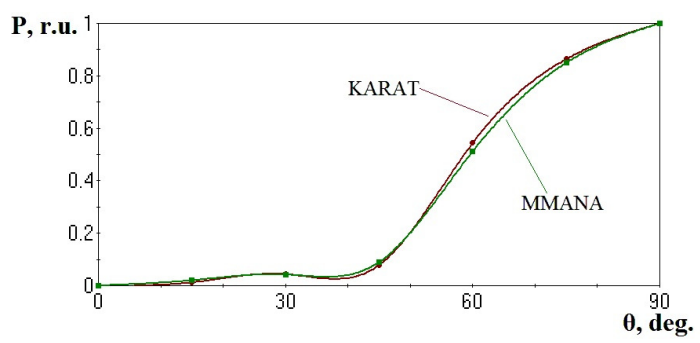

Figure 3. Radiation pattern for models of MDA in the MMANA program (green) and in the KARAT code (red).

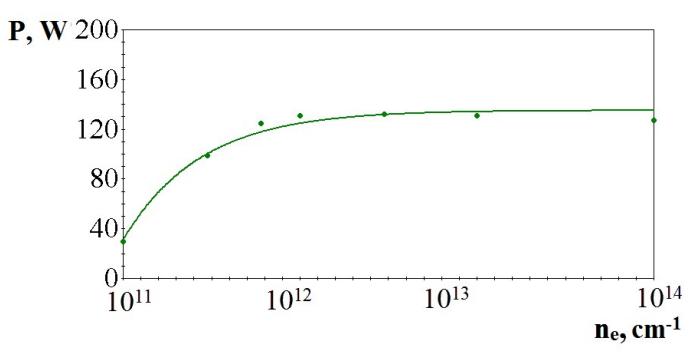

Figure 4. Radiation power $P$ from the plasma antenna vs. electron plasma density $n_{\mathrm{e}}$.

\section{Results And Discussion}

The well-known plasma dispersion equation for unmagnetized plasma is

$$
\epsilon(\omega)=\epsilon_{0}\left(1+\frac{\omega_{\mathrm{p}}^{2}}{\omega\left(\mathrm{i} \nu_{\mathrm{e}}-\omega\right)}\right),
$$

where the parameters $\omega_{\mathrm{p}}=\sqrt{n_{\mathrm{e}} e^{2} / m_{\mathrm{e}} \epsilon_{0}}, \epsilon_{0}$ is the permittivity of the free space, $n_{\mathrm{e}}$ is the plasma electron density, $e$ is the electric charge, $m_{\mathrm{e}}$ is the mass of an electron and $\nu_{\mathrm{e}}$ is the average collision frequency of the electrons. Throughout the simulation, we use the plasma density as a plasma parameter. The dependence of the radiation power on the plasma density for the plasma antenna (length $l=17 \mathrm{~cm}$, frequency $f=400 \mathrm{MHz})$ is presented in Fig. 4. The graph shows that the maximum radiation power values is for $n_{\mathrm{e}} \geq 10^{12} \mathrm{~cm}^{-1}$ and is almost constant while the density increases.

For the MDA and PDA dependences of radiation power $P$ on the length of the antenna, $l$ was calculated for various radii of the antenna (Fig. 5 ) (frequency $f=400 \mathrm{MHz}$ ). The graphs show the resonance of dependences $P$ on $l$. The resonance shifts to short waves with increasing radius $r$, because the capacity 

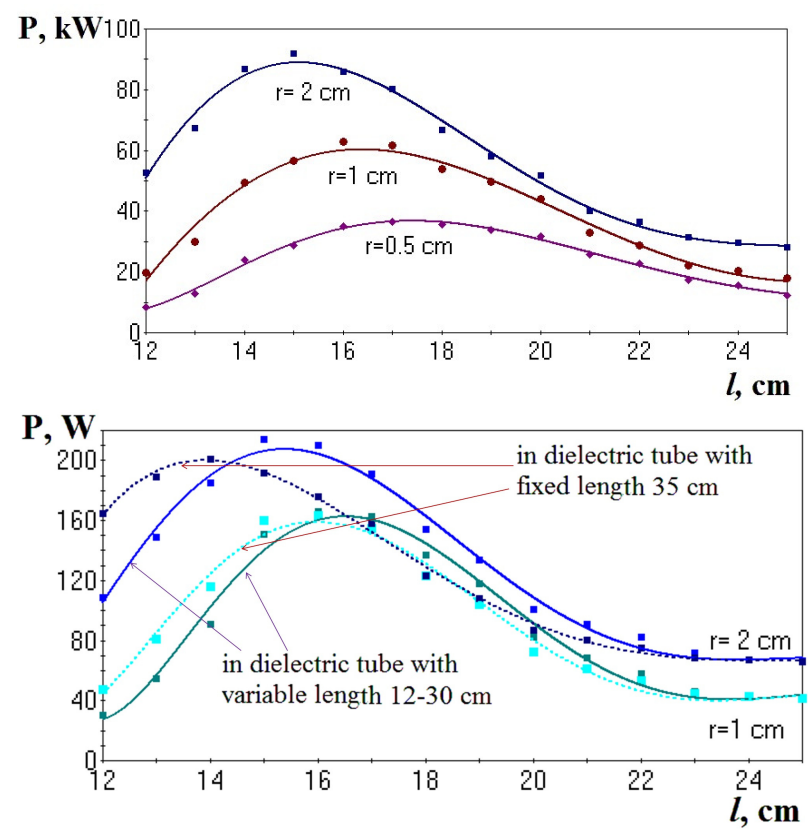

Figure 5. Radiation power $P$ of MDA (a) and PDA (b) vs. length $l$ of the antenna.

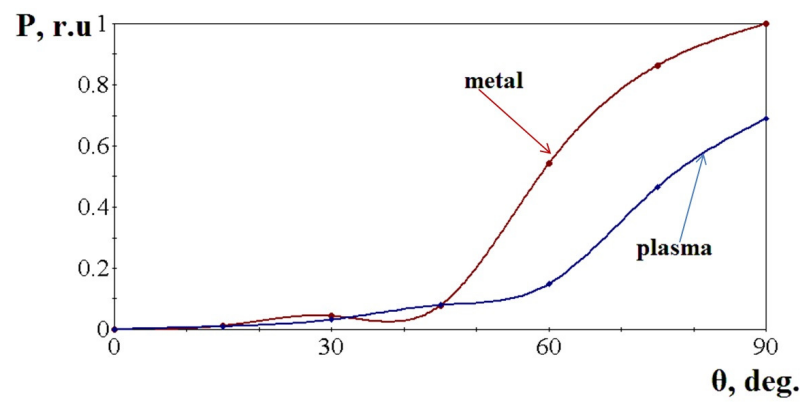

Figure 6. Radiation patterns of metal (red) and plasma (blue) vibrator antennas in KARAT code.

of the equivalent oscillatory circuit of the antenna also increases. The region of the resonance expands as the input impedance decreases. Two cases were considered for PDA: 1) a plasma column of variable length in the dielectric tube of fixed length $35 \mathrm{~cm}$; 2) a plasma column in the dielectric tube of variable length. Case 1) corresponds to an extension of the coil of the equivalent oscillatory circuit of the antenna (shift of the resonance and a weak decrease in the radiation power).

For PDA $\left(l=17 \mathrm{~cm}, r=1 \mathrm{~cm}, n_{\mathrm{e}}=3 \times 10^{12} \mathrm{~cm}^{-3}\right.$ and $f=400 \mathrm{MHz}$ ) the radiation pattern was calculated on a sphere of radius $\rho=270 \mathrm{~cm}(\approx 3 \div 4 \lambda)$. The RP of PDA and the RP of MDA (Fig. 6) are similar in form. However, the RP value for the plasma antenna is substantially smaller than the RP value for the metal antenna, because there are bigger losses for matching the coaxial cable to the antenna.

These frequency dependences of the radiation power for PDA $(l=17 \mathrm{~cm}, r=1 \mathrm{~cm})$ for various densities $n_{\mathrm{e}}$ are illustrated in Fig. 7

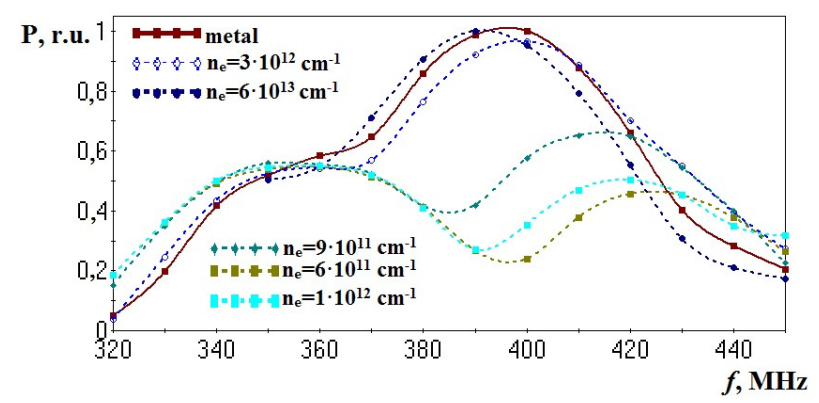

Figure 7. Radiation power $P$ of the MDA and PDA vs. the frequency.

\section{Conclusions}

The graph shows, that:

- PDA is identical with MDA when plasma density is $n_{\mathrm{e}}>2 \times 10^{12} \mathrm{~cm}^{-3}$,

- for density $10^{11}<n_{\mathrm{e}}<10^{12} \mathrm{~cm}^{-3}$, the dependences have two smooth resonances with almost the same power value.

This indicates that it may be possible to tune the working frequency with almost zero-delay and without loss of effectiveness. A change in the plasma density can control the characteristics of a plasma antenna, for example, by switching two working frequencies. This property can be used actively to create a system of phased array antennas.

\section{ACKNOWLEDGEMENTS}

The study was supported by the Ministry of Education and Science of Russia, project 8392.

\section{REFERENCES}

[1] I. Alexeff, T. Anderson, I. Farshi, et al. Experimental and theoretical results with plasma antennas. Physics of Plasmas 15:057104, 2008.

[2] I. V Goncharenko. Kompyuternoe modelirovanie antenn (Computer-aided antenna modeling). RadioSoft, Moscow, 2002. (in Russian).

[3] E. N. Istomin, D. M. Karfidov, I. M. Minaev, et al. Plasma asymmetric dipole antenna excited by a surface wave. Plasma Physics Reports 32(5):388, 2006.

[4] I. M. Minaev, N. G. Gusein-zade, K. Z. Rukhadze. A plasma receiving dipole antenna. Plasma Physics Reports 36(10):914, 2010.

[5] V. P. Tarakanov. User's Manual for Code KARAT. VA: Berkley Research Associates, Inc, Springfield, 1992. 